\title{
COVID-19-Impfungen: Replizierend oder Nichtreplizierend?
}

\author{
Der Vektor in Sputnik-V
}

Autoren

L. Klimek' , A. M. Chaker², M. Cuevas³ , S. Becker4

Institute

1 Zentrum für Rhinologie und Allergologie, Wiesbaden

2 HNO-Klinik und Zentrum für Allergie und Umwelt, TUM School of Medicine, Klinikum rechts der Isar, Technische Universität München

3 Klinik für Hals-, Nasen- und Ohrenheilkunde, Kopf- und Halschirurgie, Universität Tübingen

4 HNO-Universitätsklinik Tübingen

Schlüsselwörter

SARS-CoV-2, Sputnik V, Vektorimpfstoffe, COVID-19

Key words

SARS-CoV2, Sputnik V, Vector-Vaccines, COVID-19

online publiziert 27.05.2021

Bibliografie

Laryngo-Rhino-Otol 2021; 100: 603-607

DOI 10.1055/a-1509-8916

ISSN 0935-8943

(C) 2021. Thieme. All rights reserved.

Georg Thieme Verlag KG, Rüdigerstraße 14,

70469 Stuttgart, Germany

Korrespondenzadresse

Professor Dr. med. L. Klimek

Zentrum für Rhinologie und Allergologie Wiesbaden,

An den Quellen 10, 65183 Wiesbaden, Deutschland

Tel.: $+49 / 611 / 3086080$

Ludger.Klimek@Allergiezentrum.org

\section{ZUSAMMENFASSUNG}

Innovative und wirksame Impfstrategien entwickeln sich zum wichtigsten Hebel zur Bekämpfung der durch SARS-COV-2 ausgelösten globalen Pandemie. Innerhalb weniger Monate wurden durch Wissenschaftler weltweit vielversprechende neue Vakzine entwickelt; hierbei nutzen mehrere Impfstoffe das Prinzip adenoviraler Vektoren zum Einbringen der eigentlich immunogenen Moleküle des SARS-Coronavirus zum Auslösen einer Immunantwort. Die vom russischen Gamaleya-Institut entwickelte COVID-19-Vakzine Sputnik V (Gam-COVIDVac) nutzt die adenoviralen Vektoren 26 und 5, um das voll-

ständige SARS-Spike-Protein zur Impfung einzubringen, wobei die unterschiedlichen adenoviralen Vektoren mögliche neutralisierende Effekte gegen Adenoviren umgehen und dadurch eine ausreichende Immunogenität auch bei der Zweitimpfung (booster) gewährleisten. Die bisher veröffentlichten Studien werden teils kontrovers diskutiert, u. a. wegen kleiner Fallzahlen in Phase II und früher klinischer Endpunkte in der Phase III. Auch die bisher fehlende Verfügbarkeit der vollständigen Studienprotokolle und Datensätze wurde in der wissenschaftlichen Gemeinschaft zur Kenntnis genommen. Vulnerable Patientengruppen könnten durch eine wie in Brasilien beschriebene erhaltene Vermehrungsfähigkeit der Ad5-Adenoviren oder wie in der Slowakei beobachtete fehlende Chargenreproduzierbarkeit gefährdet werden, ein Wirksamkeitsverlust der Impfung bei Gesunden ist möglich. Die finale Bewertung in einem geordneten Zulassungsverfahren (z. B. EMA) bleibt daher abzuwarten.

\section{ABSTRACT}

Innovative and effective vaccination strategies are the most important lever to address the global SARS-COV2 pandemic. Within months scientists all over the world have developed promising new vaccines, many of which use adenoviral vectors to incorporate immunogenic molecules of SARS-coronavirus in order to elicit effective immune responses. The Gamaleya institute developed the COVID-19 vaccine named Sputnik (Gam-COVID-Vac) using adenoviral vectors ad 26 and ad5 to incorporate a full SARS-Spike Protein for vaccination. Two differing vectors enable so called prime-boost, thus avoiding neutralizing effects against the vector itself, ensuring proper immunogenicity against the vaccine. Current available published evidence has raised controversy among small sample sizes in phase II and early endpoints in phase III studies with Sputnik and scientific community took notice that full study protocols and clinical data haven't been made available yet. Patient subgroups and vaccination efficacy in healthy vaccinated may be at risk in case of partial viral replication of Ad5 vectors or when batch to batch reproducibility is not warranted, as concerns from authorities in Brazil and Slovakia have recently been raised. Final approval by governing health authorities (e. g. EMA) should therefore be awaited. 


\section{Einleitung}

Nachdem im März 2020 die Weltgesundheitsorganisation (WHO) die durch das Severe acute respiratory syndrome coronavirus type 2 (SARS-CoV-2)-Virus übertragene Coronavirus-19 (COVID-19)-Infektionskrankheit zur Pandemie erklärt hat, sind zahlreiche Positionspapiere und Handlungsempfehlungen internationaler und nationaler allergologischer Fachgesellschaften zum Umgang mit HNO-ärztlichen und allergologischen Erkrankungen und deren Therapien innerhalb und außerhalb der Pandemie publiziert worden [1-20].

Auf dieser Grundlage haben wissenschaftliche Fachgesellschaften Empfehlungen für COVID-19-Vakzinationen publiziert.

Ziel der vorliegenden Übersichtsarbeit ist es, den Einsatz des COVID-19-Impfstoffs Sputnik V für COVID-19-Vakzinierungen im Kontext aktueller Informationen darzustellen.

Der COVID-19-Impfstoff Sputnik V (Gam-COVID-Vac) ist ein auf Adenoviren basierender, 2-teiliger Impfstoff gegen das Coronavirus SARS-CoV-2. Sputnik V nutzt ein abgeschwächtes Virus (Vektor), um das SARS-CoV-2-Spike-Protein zu transportieren und eine Immunantwort zu stimulieren. Auch die Impfstoffe von AstraZeneca und Johnson \& Johnson sind Vektorimpfstoffe. Als Vektor wird hierbei ein Trägervirus bezeichnet; bei allen 3 Coronavirus-Vektorimpfstoffen handelt es sich dabei um Adenoviren, jedoch setzen alle 3 verschiedene Virusarten ein.

Sputnik V ist ein 2-Komponenten-Impfstoff, in dem die Adenovirus-Serotypen 5 und 26 verwendet werden. Das SARS-CoV-2Antigen-Insert ist ein unmodifiziertes S-Protein in voller Länge. Der Impfstoff Sputnik V wird auf der Zelllinie HEK293 hergestellt und wurde entwickelt vom staatlichen Institut Gamaleya des russischen Gesundheitsministeriums (Russian National Research Centre of Epidemiology and Microbiology). Gamaleya hat bereits einen Impfstoff gegen das Ebolavirus sowie gegen das MERS (Middle East Respiratory)-Virus entwickelt.

Sputnik V verwendet an unterschiedlichen Vektoren Typ 26 (Ad26.COV2-S) für die erste und Typ 5 (Ad5) für die zweite Impfung. Damit will man verhindern, dass sich nach der ersten Teilimpfung eine Immunantwort auf Ad26 ausbildet und damit eine Immunantwort auf den Vektor induziert wird, der die Wirksamkeit der zweiten Impfung auf SARS-CoV-2 reduzieren oder unerwünschte Wirkungen hervorrufen könnte.

Sputnik V wird vom russischen Direktinvestitionsfonds RDIF und dem Nationalen Forschungszentrum für Epidemiologie und Mikrobiologie Gamaleya entwickelt und vertrieben. Am 27. Oktober 2020 gab der RDIF bekannt, dass er bei der Weltgesundheitsorganisation einen Antrag auf eine Notfallaufnahme und Präqualifikation des Impfstoffkandidaten Sputnik V eingereicht hat.

Der Sputnik-V-Impfstoff wird durch eine 4-stufige Reinigung (2 Chromatografie-Stufen und 2 Tangentialflussfiltrations-Stufen) hergestellt. In den Herstellerinformationen wird angegeben, dass bei der Sputnik-V-Impfstoffproduktion nur nichtreplizierende adenovirale Vektoren vom Typ E1 und E3 verwendet werden, die auf HEK293-Zellen entwickelt und produziert werden.

Denis Logunov und Kollegen berichteten am 2. Februar 2021 in The Lancet über die Zwischenergebnisse einer Phase-III-Studie mit dem Impfstoff Sputnik V COVID-19. Die Studienergebnisse zeigen eine konsistent starke Schutzwirkung über alle Altersgruppen der Teilnehmer hinweg [21].

Gam-COVID-Vac verwendet einen heterologen rekombinanten Adenovirus-Ansatz mit dem Adenovirus 26 (Ad26) und Adenovirus 5 (Ad5) als Vektoren für die Expression des Spike-Proteins. Durch die Verwendung von 2 unterschiedlichen Serotypen, die im Abstand von 21 Tagen verabreicht werden, soll eine eventuell bereits bestehende Adenovirus-Immunität in der Bevölkerung ausgeschaltet werden [22]; ein ähnlicher Ansatz wurde bereits bei der Entwicklung eines Ebolavirus-Impfstoffs angewendet [23] und als Prinzip des Prime Boosts mit 2 verschiedenen Vektoren bezeichnet [24]. Der rekombinante Adenovirus-Ansatz wird auch beim in Oxford entwickelten Impfstoff von AstraZeneca verwendet, der ein Schimpansen-Adenovirus (ChAdOx) nutzt [25], sowie beim Impfstoff Ad266 von Johnson \& Johnson [26] und beim Impfstoff auf Ad5-Basis von CanSinoBIO-Beijing [27].

Die Trägerviren sollen so modifiziert worden sein, dass sie nicht vermehrungsfähig sind und keine Infektion auslösen können. Sie werden in die humanen Zellen aufgenommen, exprimieren das SARS-CoV-2-Spike-Protein und werden dann inaktiviert und abgebaut, auch wenn einige Adenovirus-Gene exprimiert nachgewiesen werden konnten [28]. Die mit dem Impfstoff infizierten Zellen werden schließlich durch genau die Immunmechanismen zerstört, die der Impfstoff ausgelöst hat.

Rekombinante Adenoviren sind als Impfstoffvektoren gut geeignet, da sie große genetische „Nutzlasten“ aufnehmen können und das angeborene Immunsystem aktivieren [29]. Folglich benötigen sie kein Adjuvans und können teilweise bereits nach einer einzigen Dosis Immunität vermitteln [24]. Ihre physikalische Robustheit erlaubt eine Lagerung bei Temperaturen um $-18^{\circ} \mathrm{C}$, was für viele Lieferketten (Pharma-Großhandel) praktikabel ist. Der Nachteil rekombinanter Adenovirus-basierter Impfstoffe besteht darin, dass vergleichsweise große Dosierungen und viele Partikel benötigt werden, was hohe Anforderungen an die Herstellung und Quantifizierung stellt.

Klinische Daten zum Impfstoff Sputnik V wurden zunächst als frühe Phase-I/II-Daten im September 2020 veröffentlicht [30]. Die Empfänger generierten demnach deutliche Antikörperantworten gegen das SARS-CoV-2-Spike-Protein, darunter auch neutralisierende Antikörper, die die Bindung des Virus an seinen Rezeptor hemmen. Auch Hinweise auf T-Zell-Antworten wurden dargestellt und bei keinem Teilnehmer seien schwere Nebenwirkungen aufgetreten [30].

Allerdings nahmen lediglich jeweils 38 Probanden an den 2 Studien zur Sicherheit, Verträglichkeit und Immunogenität teil. Diese Ergebnisse stießen nicht nur wegen der kleinen Testgruppe auf starke Vorbehalte. Einer Gruppe internationaler Immunologen waren zudem Merkwürdigkeiten aufgefallen [31]. Diese Autoren berichten, dass die verschiedenen Probanden zwar ganz unterschiedliche Formen des Impfstoffs bekamen, sie aber laut der Studienergebnisse an verschiedenen Tagen alle den exakt gleichen Antikörper-Titer im Blut hatten [31]. Dass zudem bei einigen Probanden auch die Anzahl SARS-CoV-2-reaktiver T-Zellen identisch war, kann nach Ansicht dieser internationalen Expertengruppe nicht durch zufällige Ereignisse erklärt werden [31]. In einem offenen Brief stellte eine große Gruppe von Forschenden aus Europa, 
den USA, Kanada, aber auch aus Russland mögliche Datenmanipulationen als Erklärung für diese Auffälligkeiten zur Diskussion [31].

Das russische Forscherteam hinter Sputnik V wies die Bedenken in einer Correspondence in der Zeitschrift Lancet zurück und wies darauf hin, dass einige der angeblichen Unregelmäßigkeiten, wie z. B. verdächtig ähnliche Antikörperzahlen unter den Teilnehmern, wahrscheinlich Zufälle gewesen seien, teilweise verursacht durch Faktoren wie die geringe Stichprobengröße und die Rundung von Zahlen in den verschiedenen Datenpunkten [32].

Nachfolgend wurde am 02.02.2021 ein Zwischenbericht zu den laufenden Phase-III-Studien publiziert [21], der die Ergebnisse von mehr als 20000 Teilnehmern darstellte, von denen $75 \%$ den Impfstoff erhielten.

Demnach bekamen mehr als 18000 Probanden jeweils 2 Dosen des Impfstoffs im Abstand von 3 Wochen geimpft. Bei einer geplanten Studienpower von $85 \%$ waren die rekrutierten Teilnehmer 18 Jahre und älter und zu etwa $60 \%$ männlich. Komorbiditäten, die ein bekanntes Risiko für den Schweregrad von COVID-19 darstellen, waren bei etwa einem Viertel der Studienteilnehmer vorhanden. Bei $n=62$ (1,3\%) von 4902 Personen in der Placebogruppe und $n=16(0,1 \%)$ von 14964 Teilnehmern in der Impfstoffgruppe wurde eine SARS-CoV-2-Infektion ab Tag 21 nach der ersten Impfstoffdosis bestätigt (was als primärer Endpunkt vorbestimmt worden war).

Eine zeitaufgelöste Darstellung der Inzidenzrate in den beiden Gruppen zeigte, dass die zur Verhinderung der Erkrankung erforderliche Immunität innerhalb von 18 Tagen nach der ersten Dosis eintrat. Drei Todesfälle traten in der Impfstoffgruppe bei Personen mit umfangreichen Komorbiditäten auf und wurden als nicht im Zusammenhang mit dem Impfstoff stehend angesehen. Es wurden keine schwerwiegenden unerwünschten Ereignisse registriert, die als mit dem Impfstoff in Verbindung stehend angesehen wurden, aber schwerwiegende unerwünschte Ereignisse, die nicht mit dem Impfstoff in Verbindung standen, wurden bei 45 Teilnehmern aus der Impfstoffgruppe und 23 Teilnehmern aus der Placebogruppe berichtet. Die Wirksamkeit des Impfstoffs, basierend auf der Anzahl der bestätigten COVID-19-Fälle ab 21 Tagen nach der ersten Impfdosis, wird mit 91,6\% (95\%-KI 85,695,2 ) angegeben.

Auch diese Publikation stieß unter Impfexperten auf Kritik, da eine fehlende Veröffentlichung der Primärdaten eine unabhängige Bewertung unmöglich macht [33]. Zudem wurde darauf hingewiesen, dass z. B. der primäre Endpunkt zu einem ungewöhnlich frühen Zeitpunkt analysiert wurde (21 Tage nach der ersten Dosis und nicht nach der zweiten Dosis). Das Originalprotokoll für die Studie wurde ebenfalls nicht veröffentlicht, sodass es nicht möglich ist zu erkennen, ob dies vor oder während der Studie entschieden wurde [34].

\section{Können die als Vektor benutzten Adenoviren replizieren? Brasilianische Gesundheitsbehör- de warnt und zieht Konsequenzen}

Sputnik V verwendet aus oben dargelegten Gründen 2 verschiedene Vektoren: Ad26.COV2-S für die erste und Typ 5 (Ad5) für die zweite Impfung. Die Vektoren dienen dem Transport des Spike-Proteins von SARS-CoV-2 an und in die humanen Zellen. Die bei Vektorimpfstoffen als Vektoren gewählten Adenoviren sind in der Regel harmlose Erkältungsviren oder nichthumanpathogene Viren. Sie werden zudem inaktiviert, indem man in der Regel die Proteine E1 und E3 aus dem Adenovirus herausschneidet. Damit wird auch Platz für die Aufnahme des Spike-Proteins geschaffen.

Im Rahmen der Zulassungsuntersuchungen für Sputnik V durch die brasilianische Gesundheitsbehörde Anvisa wurde nun darüber berichtet, dass der Vektor Ad5 nicht inaktiviert gewesen sei und vermehrungsfähig war [35]. Bei einem Test mit kultivierten Lungenepithelzellen (A549-Zellen) bildete Sputnik V Plaques aus, was auf eine Infektion mit Ad5-Adenoviren zurückzuführen gewesen sei [35]. Als Grund hierfür wird eine unzureichende Entfernung von E1 angenommen, wodurch die Ad5-Adenoviren replikationsfähig (vermehrungsfähig) waren, und es wird spekuliert, ob hierdurch überdies nicht ausreichend Platz für das SARS-CoV2-Spike-Protein vorhanden war, weshalb eine unzureichende Immunantwort gegen SARS-CoV-2 befürchtet wird [35]. Diese Replikationsfähigkeit wurde in allen den brasilianischen Behörden zur Prüfung eingereichten Chargen von Sputnik V gefunden [35].

\section{Diskussion}

Der russische Impfstoff Sputnik V ist derzeit in mehreren Ländern zur Zulassung eingereicht, u. a. prüft die Europäische Arzneimittelagentur (EMA) den Impfstoff für eine EU-Zulassung [36]. Für Klarheit in Bezug auf Wirksamkeit und Sicherheit soll ein RollingPreview-Verfahren der EMA mit dem Ziel einer EU-weiten Zulassungsempfehlung sorgen, auch hierfür braucht die EMA allerdings den vollständigen Original-Datensatz, der bislang aus Russland nach Angaben verfügbarer Quellen wohl noch nicht zur Verfügung gestellt wurde [37, 38].

„Die EMA ist gesetzlich verpflichtet zu überprüfen, ob Hersteller von Arzneimitteln, für die in der EU eine Marktzulassung beantragt wird, die Good Manufacturing Practice (GMP) einhalten “, so die Antwort der Behörde auf eine entsprechende Anfrage [38].

Bereits im März hatte Emer Cook, Direktorin der EMA, eine Inspektion von Produktionsstätten des Impfstoffs angekündigt. Auch Kliniken, in denen Sputnik V klinisch getestet wurde, sollen besucht werden, um die Einhaltung der Good Clinical Practice (GCP) zu prüfen [38]. „Klinische Studien, die für eine EU-Marktzulassung vorgelegt werden, müssen der GCP entsprechen. Die Einhaltung des Standards gewährleistet, dass die Rechte, die Sicherheit und das Wohlbefinden der Studienteilnehmer geschützt werden und dass Daten aus klinischen Studien glaubwürdig sind“, so die EMA [38].

Ein solches Vorgehen ist durchaus normal und wird auch bei anderen Impfstoffen und Medikamenten angewendet. Innerhalb der EU wird die Einhaltung der Standards für die Arzneimittelproduktion von den nationalen Regulierungsbehörden überwacht, und die EMA koordiniert diese Arbeit. Außerhalb der EU darf eine Inspektion nur dann unterlassen werden, wenn sie bereits in den letzten 2 oder 3 Jahren durchgeführt wurde.

Die Ablehnung des Impfstoffs durch die brasilianische Gesundheitsbehörde Anvisa kann in diesem Gesamtzusammenhang nun 
aber als sehr bedeutsam angesehen werden. In Brasilien hatten mehrere Bundesstaaten und das Gesundheitsministerium zusammen ca. 76 Millionen Sputnik-V-Dosen bestellt.

Zum Zeitpunkt der Erstellung dieser Publikation hatten außer Russland ca. 60 Staaten auf der ganzen Welt (u. a. Argentinien, Indien, Mexiko, Iran, Ghana, Sri Lanka, Montenegro oder Serbien) Sputnik V für den Notfalleinsatz zugelassen, darunter auch Ungarn und die Slowakei als bislang einzige EU-Länder [34]. Ungarn und die Slowakei haben den Einsatz von Sputnik V jeweils per nationaler Notfallzulassung erlaubt. In Ungarn wird das Vakzin tatsächlich schon verimpft, während in der Slowakei 200000 am 1. März von Russland gelieferte Sputnik-Dosen nicht von den Behörden freigegeben wurden [38]. Die Slowakei hatte eine Ausnahmegenehmigung für die Anwendung von Sputnik V erteilt, jedoch den tatsächlichen Einsatz von einer Prüfung durch das slowakische Institut für Arzneimittelkontrolle (SUKL) abhängig gemacht, das jedoch Zweifel an der Sicherheit der erhaltenen Charge hatte: Sie habe „nicht die gleichen Merkmale und Eigenschaften“ wie die Chargen, deren klinische Testergebnisse in „The Lancet“ veröffentlicht worden waren [38]. Der Hersteller habe trotz wiederholter Anfragen keine Daten aus klinischen Studien oder der Produktion geliefert [38].

Sputnik V wird außerhalb Russlands zu einem Preis von ca. $€ 8,40$ pro Dosis für internationale Käufer angeboten, laut dem russischen Hersteller RDIF wurden Verträge mit mehr als einem Dutzend Herstellern in 10 Ländern unterzeichnet, um insgesamt 1,4 Milliarden Dosen zu produzieren [34].

In Russland selbst wurden laut dem Statistikportal Our World in Data bis zum 2. Mai allerdings nur ca. 20 Millionen Dosen von Sputnik V verimpft, ca. 8,6\% der Bevölkerung haben die erste Impfdosis erhalten, was im internationalen Vergleich eher wenig ist [39].

\section{Fazit}

Sollte sich die Replikationsfähigkeit für den Vektor Ad5 in Sputnik V in weiteren unabhängigen Untersuchungen bestätigen, würden wir davon ausgehen, dass der Impfstoff in dieser Form in der EU nicht zulassungsfähig wäre. Hierbei kann diskutiert werden, ob es sich um ein Problem in der Impfstoffentwicklung und/oder der Herstellung handelt.

Die Vermehrungsfähigkeit der Ad5-Adenoviren ist klinisch in erster Linie für bestimmte Patientengruppen ein Problem (immunsupprimierte und ältere Patienten, Patienten mit Vorerkrankungen und Schwangere). Entscheidender könnte der Wirksamkeitsverlust in der Impfwirkung gegen SARS-COV-2 sein, vor allem aber der Vertrauensverlust in einen vielversprechenden Impfstoff, der in diesem Fall nicht den angegebenen Spezifikationen entsprechen würde und bei welchem die Angaben zu Effektivität und Sicherheit derzeit ebenfalls zur Disposition gestellt sind.

\section{Interessenkonflikt}

Die Autorinnen/Autoren geben an, dass kein Interessenkonflikt besteht.

\section{Literatur}

[1] Andriollo G, Urbani S, Buonomo A et al. Rapid protocol for irinotecan desensitization: a case report and literature review. Allergo Journal International 2020; 29: 286-288. doi:10.1007/s40629-020-00156-0

[2] Ankermann T, Spindler T, Gerstlauer M et al. CME. Allergie und Impfen ein Mythos entmystifiziert. Allergies and vaccination: a myth demystified. Allergo J Int 2018; 27: 234-243. doi:317060 de-38m

[3] Bangerl T, Zahel B, Lueger A et al. Hypersensitivity reactions to non-steroidal anti-inflammatory drugs: results of an Austrian cohort study. Allergo Journal International 2020; 29: 227-232. doi:10.1007/s40629020-00134-6

[4] Bork K, Aygören-Pürsün E, Bas M et al. Guideline: Hereditary angioedema due to C1 inhibitor deficiency. Allergo Journal International 2019; 28 : 16-29. doi:10.1007/s40629-018-0088-5

[5] Bradfisch F, Pietsch M, Forchhammer S et al. Anaphylaktische Reaktionen nach Tollwutimpfungen mit Sensibilisierung gegenüber Gelatine Fallserie und Literaturübersicht. Allergo J Int 2019; 28: 103-106 Case series of anaphylactic reactions after rabies vaccinations with gelatin sensitization. Allergo-Journal 2019; 28: 18. doi:317060 de-38m

[6] Can Bostan Ö, Cakmak M, Kaya S et al. Anaphylaxis to lidocaine and crossreactivity to articaine and prilocaine with tolerance to bupivacaine. Allergo Journal International 2020; 29: doi:10.1007/s40629-020-00132-8

[7] Gülsen A, Wedi B, Jappe U. Hypersensitivity reactions to biologics (part I): allergy as an important differential diagnosis in complex immune-derived adverse events. Allergo Journal International 2020; 29: 97-125. doi:10.1007/s40629-020-00126-6 doi

[8] Gülsen A, Wedi B, Jappe U. Hypersensitivity reactions to biologics (part II): classifications and current diagnostic and treatment approaches. Allergo Journal International 2020; 29: 139-154. doi:10.1007/s40629020-00127-5

[9] Herz A, Kopp MV. Anaphylactic reaction at a pizzeria in a 13-year-old female patient. Allergo Journal International 2020; 29: 165-167. doi:10.1007/s40629-020-00123-9

[10] Honda T, Kuriyama K, Kiso K et al. Incidence rate of severe adverse drug reactions to nonionic contrast media at the National Hospital Organization Osaka National Hospital. Allergo Journal International 2020; 29: 240-244. doi:10.1007/s40629-020-00139-1

[11] Klimek L, Bergmann KC, Brehler $R$ et al. Practical handling of allergic reactions to COVID-19 vaccines: A position paper from German and Austrian Allergy Societies AeDA, DGAKI, GPA and ÖGAI. Allergo Journal International 2021. doi:10.1007/s40629-021-00165-7

[12] Klimek L, Chaker AM, Cuevas M. Allergische Reaktionen auf COVID-19Impfungen - Was HNO-Ärzte wissen sollten - Teil 1: Immunologische Grundlagen von Allergien auf Impfstoffe, Immunmechanismen von allergischen und pseudoallergischen Reaktionen; Teil 2: Charakteristika der mRNA-Impfstoffe BNT162b2- und mRNA-1273 zur Prophylaxe von COVID-19 und assoziierte Immunphänomene; Teil 3: Praktische Aspekte der Prophylaxe, Diagnostik und Therapie von Allergien auf COVID-19Impfstoffe. Laryngorhinootologie 2021; 100: 344-354

[13] Klimek L, Novak N, Hamelmann E et al. Severe allergic reactions after COVID-19 vaccination with the Pfizer/BioNTech vaccine in Great Britain and USA: Position statement of the German Allergy Societies: Medical Association of German Allergologists (AeDA), German Society for Allergology and Clinical Immunology (DGAKI) and Society for Pediatric Allergology and Environmental Medicine (GPA). Allergo Journal International 2021; 30: 51-55. doi:10.1007/s40629-020-00160-4

[14] Klimek L, Worm M, Lange L et al. Management von Anaphylaxie-gefährdeten Patienten während der Covid-19-Pandemie. Allergo Journal International 2020; 29: 16-26. doi:10.1007/s15007-020-2618-y

[15] Mickler M, Drexler K, Schreml S. Wheat-dependent exercise-induced anaphylaxis with Tri-a-14-sensitization as part of a lipid transfer protein 
syndrome. Allergo Journal International 2020; 29: 9-11. doi:10.1007| s40629-019-0091-5

[16] Mohamed Khazin S, Abdullah D, Liew AKC et al. IgE-mediated hypersensitivity to chlorhexidine among first-year dental students. Allergo Journal International 2019; 28: 204-208. doi:10.1007/s40629-019-0103-5

[17] Paulmann M, Mockenhaupt M. Severe skin reactions: clinical picture, epidemiology, etiology, pathogenesis, and treatment. Allergo Journal International 2019; 28: 311-326. doi:10.1007/s40629-019-00111-8

[18] Querbach C, Biedermann T, Busch DH et al. Suspected penicillin allergy: risk assessment using an algorithm as an antibiotic stewardship project. Allergo Journal International 2020; 29: 174-180. doi:10.1007/s40629020-00135-5

[19] Ring J, Beyer K, Biedermann T et al. Guideline (S2k) on acute therapy and management of anaphylaxis: 2021 update: S2k-Guideline of the German Society for Allergology and Clinical Immunology (DGAKI), the Medical Association of German Allergologists (AeDA), the Society of Pediatric Allergology and Environmental Medicine (GPA), the German Academy of Allergology and Environmental Medicine (DAAU), the German Professional Association of Pediatricians (BVKJ), the Society for Neonatology and Pediatric Intensive Care (GNPI), the German Society of Dermatology (DDG), the Austrian Society for Allergology and Immunology (ÖGAI), the Swiss Society for Allergy and Immunology (SGAI), the German Society of Anaesthesiology and Intensive Care Medicine (DGAI), the German Society of Pharmacology (DGP), the German Respiratory Society (DGP), the patient organization German Allergy and Asthma Association (DAAB), the German Working Group of Anaphylaxis Training and Education (AGATE). Allergo Journal International 2021; 30: 1-25. doi:10.1007/s40629-020-00158-y

[20] Wurpts G, Aberer W, Dickel H et al. Guideline on diagnostic procedures for suspected hypersensitivity to beta-lactam antibiotics. Allergo Journal International 2019; 28: 121-151. doi:10.1007/s40629-019-0100-8

[21] Logunov DY, Dolzhikova IV, Shcheblyakov DV et al. Safety and efficacy of an rAd26 and rAd5 vector-based heterologous prime-boost COVID-19 vaccine: an interim analysis of a randomised controlled phase 3 trial in Russia. Lancet (London, England) 2021; 397: 671-681. doi:10.1016/ S0140-6736(21)00234-8

[22] Barouch DH, Kik SV, Weverling G] et al. International seroepidemiology of adenovirus serotypes 5, 26, 35, and 48 in pediatric and adult populations. Vaccine 2011; 29: 5203-5209. doi:10.1016/j.vaccine.2011.05.025

[23] Dolzhikova IV, Zubkova OV, Tukhvatulin Al et al. Safety and immunogenicity of GamEvac-Combi, a heterologous VSV- and Ad5-vectored Ebola vaccine: An open phase I/II trial in healthy adults in Russia. Human vaccines \& immunotherapeutics 2017; 13: 613-620. doi:10.1080/ 21645515.2016.1238535

[24] Lu S. Heterologous prime-boost vaccination. Current opinion in immunology 2009; 21: 346-351. doi:10.1016/j.coi.2009.05.016

[25] Voysey M, Clemens SAC, Madhi SA et al. Safety and efficacy of the ChAdOx1 nCoV-19 vaccine (AZD1222) against SARS-CoV-2: an interim analysis of four randomised controlled trials in Brazil, South Africa, and the UK. Lancet (London, England) 2020; 397: 99-111. doi:10.1016/ S0140-6736(20)32661-1

[26] Sadoff J, Le Gars M, Shukarev G et al. Safety and immunogenicity of the Ad26.COV2.S COVID-19 vaccine candidate: interim results of a phase 1/ 2a, double-blind, randomized, placebo-controlled trial. MedRxiv (preprint) 2020. doi:10.1101/2020.09.23.20199604

[27] Zhu FC, Guan XH, Li YH et al. Immunogenicity and safety of a recombinant adenovirus type-5-vectored COVID-19 vaccine in healthy adults aged 18 years or older a randomised, double-blind, placebo-controlled, phase 2 trial. The Lancet 2020; 396: 479-488. doi:10.1016/s01406736(20)31605-6

[28] Almuqrin A, Davidson AD, Williamson MK et al. SARS-CoV-2 vaccine ChAdOx1 nCoV-19 infection of human cell lines reveals low levels of viral backbone gene transcription alongside very high levels of SARS-CoV-2 S glycoprotein gene transcription. Genome Medicine 2021; 13: 43 doi:10.1186/s13073-021-00859-1

[29] Liu J, Ewald BA, Lynch DM et al. Magnitude and phenotype of cellular immune responses elicited by recombinant adenovirus vectors and heterologous prime-boost regimens in rhesus monkeys. Journal of virology 2008; 82: 4844-4852. doi:10.1128/JVI.02616-07

[30] Logunov DY, Dolzhikova IV, Zubkova OV et al. Safety and immunogenicity of an rAd26 and rAd5 vector-based heterologous prime-boost COVID-19 vaccine in two formulations two open, non-randomised phase 1/2 studies from Russia. The Lancet 2020; 396: 887-897. doi:10.1016/s0140-6736(20)31866-3

[31] Bucci E. Note of concern. In. Cattivi Scienziati: Cattivi Scienziati-fighting bad and pseudo-science. 2020

[32] Logunov DY, Dolzhikova IV, Tukhvatullin Al et al. Safety and efficacy of the Russian COVID-19 vaccine: more information needed - Authors' reply. Lancet (London, England) 2020; 396: e54-e55. doi:10.1016/ S0140-6736(20)31970-X

[33] Cohen J. Russia's claim of a successful COVID-19 vaccine doesn't pass the 'smell test,' critics say. SienceMag.org: SienceMag.org 2020. doi:10.1126/science.abf6791

[34] Baraniuk C. Covid-19: What do we know about Sputnik V and other Russian vaccines? BMJ (Clinical research ed) 2021; 372: n743 doi:10.1136/bmj.n743

[35] Vilela P. Brazil drug regulator rejects import and use of Sputnik V. In. AgenciaBrasil: Pedro Rafael Vilela. 2021

[36] EMA. Clarification on Sputnik V vaccine in the EU approval process. In ema.europa.eu: European Medicines Agency. 2021

[37] EMA. EMA starts rolling review of the Sputnik V COVID-19 vaccine. In. ema.europa.eu: European Medicines Agency. 2021

[38] Sheiko I. EU nimmt Sputnik V unter die Lupe. In. dw.com: ( 2021 Deutsche Welle. 2021

[39] OurWorldinData. Coronavirus (COVID-19) Vaccinations. In. Our World in Data: Our World in Data - Statistics and Research. 2021 